\title{
e-Migrinter
}

$18 \mid 2019$

L'ethnographie en migration(s)

\section{Chercheure à l'intersection de ses appartenances ethniques et de genre}

Ethnographie dans un quartier de la « maghrébinité » montréalaise

Bochra Manaï

\section{OpenEdition}

Journals

Édition électronique

URL : https://journals.openedition.org/e-migrinter/1737

DOI : 10.4000/e-migrinter. 1737

ISSN : 1961-9685

Éditeur

UMR 7301 - Migrinter

Référence électronique

Bochra Manaï, « Chercheure à l'intersection de ses appartenances ethniques et de genre », e-Migrinter [En ligne], 18| 2019, mis en ligne le 12 septembre 2019, consulté le 20 mai 2021. URL : http:// journals.openedition.org/e-migrinter/1737 ; DOI : https://doi.org/10.4000/e-migrinter.1737

Ce document a été généré automatiquement le 20 mai 2021.

Tous droits réservés 


\section{Chercheure à l'intersection de ses appartenances ethniques et de genre}

Ethnographie dans un quartier de la «maghrébinité » montréalaise

\section{Bochra Manaï}

1 L'immigration à Montréal est constitutive de l'identité de la ville. D'ailleurs, la ville fait l'objet d'une littérature prolifique sur les groupes ethniques, les espaces de l'immigration, les lieux de résidence et les trajectoires de l'immigration (Germain, 2013). Qu'est-ce qui pousse alors encore aujourd'hui à porter un intérêt sur l'objet migratoire et ethnique à Montréal ? La réalité des flux migratoires n'appartient pas au passé, mais continue bel et bien de s'inscrire dans les sociétés urbaines en modifiant les relations sociales. En prenant comme objet de recherche autant l'immigration que les interactions avec la société d'accueil, cet article a pour objectif de décrire le cheminement qui a mené à s'intéresser aux « Maghrébins » comme sujet, et ce afin de comprendre la société montréalaise dans laquelle ils ont décidé de s'inscrire. À travers cette recherche sur la "maghrébinité », se lit aussi la position du chercheur. Par " maghrébinité », nous entendons les marqueurs visibles et invisibles dont les individus font usage pour évoquer leurs appartenances ethniques, culturelles, linguistiques, religieuses etc. et en référence à la zone d'Afrique dite « du Nord ».

2 Travailler sur cette immigration maghrébine, récente au Québec, puisque les vagues migratoires ne deviennent importantes qu'à partir des années 1990 et 2000, impose de rappeler qu'elle est constituée principalement de contingents de travailleurs qualifiés. Loin de l'image de l'immigration comme phénomène exclusivement masculin, la massification de l'immigration maghrébine s'est accompagnée d'une immigration féminine importante. En 2001, près de la moitié des immigrants maghrébins sont des femmes (Manaï, 2018). En effet, les migrations internationales incluent des femmes qui deviennent elles-mêmes porteuses de projets migratoires (Morokvasic, 2008). La littérature sur les femmes maghrébines concerne souvent la réalité des espaces européens, mais ces données ne s'appliquent pas aux femmes maghrébines venues 
s'installer au Québec (Ben Soltane, 2015). Leur migration en Europe s'est traduite par une perspective "familiale " alors qu'au Québec, elle illustre un type de recrutement favorisant des immigrantes qualifiées et diplômées. Les études de certains groupes de migrants historiques installés au Québec, tels que les Italiens ou les Portugais, montraient déjà une présence féminine à analyser autant comme acteur social intracommunautaire que comme agent économique prenant le contre-pied de la figure paradigmatique de la femme "victime" dans de nombreuses recherches sur les migrations féminines. Le cas du Petit-Maghreb au Québec démontre le rôle majeur des femmes maghrébines dans leur migration, comme initiatrices de stratégies économiques (Morokvasic, 2008).

Dans son apport théorique sur les paradoxes de l'immigration, Sayad (1999) a invité au questionnement et à la réflexivité dès qu'il s'agit de se pencher sur l'altérité. Si le chercheur peut se doter de méthodes pour tenter d'atteindre une certaine objectivité, il lui est nécessaire de se doter d'une éthique et d'une réflexivité, tout au long de la recherche, notamment ethnographique. Si Sayad a révolutionné le regard sur les immigrants et la recherche sur l'ethnicité en étant lui-même partie prenante de cette trajectoire, c'est bien parce qu'il pouvait comprendre les détails majeurs et les conditions de vie quotidienne des migrants algériens. De plus, les terrains réalisés par Sayad lui étaient proches du point de vue du genre. Pour une chercheure, l'accessibilité à des terrains de recherche composés principalement par des hommes n'est pas chose aisée, même pour étudier des "sujets " qui nous ressemblent du point de vue de la trajectoire ethnique ou migratoire. Comment se présenter alors sur un terrain qui semblerait favoriser la présence des hommes? Comment faire avec des lieux d'observation qui viennent déstabiliser le confort identitaire de chercheure ? Comment observer lorsque la chercheure est elle-même le genre « absent » de ces lieux ? Lorsque les femmes sont absentes des espaces de sociabilité, n'est-ce pas l'ethnicité qui semble rapprocher la chercheure de son terrain ? C'est ce que l'on développera ici, en prenant pour illustration une recherche doctorale menée entre 2010 et 2014 dans un espace urbain montréalais désormais appelé le Petit-Maghreb. La nature de cet espace urbain a déjà été analysée (Manaï, 2018 ; 2015 ; 2014) même si l'on reviendra sur une proposition de définition. On mènera donc ici une perspective qui s'apparente à une socio-analyse (Perez, 2009) et qui permettra, en fait, de déterminer les efforts de réflexivité qu'un tel terrain a nécessité, non seulement en terme de genre mais également en terme d'ethnicité et de parcours migratoire. En questionnant la place du chercheur sur son terrain, cet article analyse la position du chercheur dit de l'intérieur et l'ambivalence que recèle la légitimité que lui conférerait ses appartenances.

\section{Dépasser les ambivalences du chercheur de l'intérieur}

4 Durant mes précédentes recherches réalisées sur l'immigration et l'ethnicité, une mise à distance constante était appliquée à certains sujets d'étude. Le refus, pour un chercheur dit co-ethnique, de travailler sur l'objet des Maghrébins, des Arabes ou des Musulmans, était une règle de travail, car le lien entre l'objet et le chercheur me semblait teinté. L'objectivité ne me semblait possible qu'à travers l'évitement du biais du co-ethnique. Parallèlement à la fonction de chercheure, une implication militante sur des questions de politique tunisienne et de défense des droits, basée sur ma trajectoire personnelle et familiale, m'a permis d'accéder au terrain sur les Maghrébins 
de Montréal dès 2009. Lorsqu'en 2011, avec l'arrivée du "Printemps arabe ", se déploient de nombreuses actions, avec des citoyens actifs et des membres de groupes arabes, maghrébins, tunisiens, algériens, marocains, issus de multiples organisations, la question de l'objectivité face au terrain s'est posée autrement. Quelle place accorder à la proximité avec la militance ? Est-il possible d'être une chercheure et une militante ? Les tergiversations d'ordre éthique devenaient au fur et à mesure une évidence : il fallait faire ce terrain parce que l'accessibilité linguistique et la connaissance des réalités méditerranéennes et françaises étaient un atout et non pas une contrainte.

5 Ce sont la spécificité du chercheur et son éthique dans la situation, notamment grâce à son positionnement réflexif, qui amènent un regard unique. Le terrain a donc été abordé comme le préconisent Daniel Cefaï et Valérie Amiraux :

«Plutôt que de se laisser prendre par surprise, mieux vaut travailler sur soi et s'engager dans une interrogation socio-analytique, comme Jean-Claude Passeron et, sur le tard, Pierre Bourdieu, nous y invitent. Ce qui risque de prendre le tour d'une interrogation obsessionnelle, d'une phobie méthodologique ou d'une répétition névrotique, une fois analysé, se transforme en atout de compréhension. » (Cefaï et Amiraux, 2002 : p. 4).

6 Cet objet de recherche a nécessité une approche prudente et minutieuse continue durant quatre années. En effet, le lien entre le chercheur et son objet d'étude a été un questionnement dès le début de ce travail. Sans que ce terrain ne soit considéré comme sensible au sens de Cefaï et Amiraux (2002), comme un terrain de guerre ou un terrain dangereux, il constituait un terrain complexe pour une chercheure femme aux origines maghrébines. L'intérêt porté à l'objet migratoire, s'il est basé sur la comparaison, permet de développer des recherches porteuses de réalités nuancées, et permet surtout de ne pas enfermer l'objet de recherche " ethnique » dans une vision essentialisante. La comparaison systématique avec d'autres espaces urbains, du contexte montréalais ou de contextes différents, tels que les espaces urbains des villes françaises ou belges, a permis de mieux comprendre la spécificité du lieu : celle d'une construction ethnique, insérée dans le contexte urbain montréalais, lui-même historiquement marqué par une fabrique de l'ethnicité visible dans la ville. En plus d'une compilation documentaire, cartographique et statistique des données sur les Maghrébins, dans leur ensemble et dans leurs sous-catégories de Tunisiens, d'Algériens ou de Marocains, le travail a consisté en une ethnographie de la "maghrébinité » montréalaise. Cette phase de travail, comprenant des observations et des entrevues, avait pour but de narrer la perspective des multiples acteurs du Petit-Maghreb, en décrivant ce qui a fait sens pour que les lieux puissent prendre forme en tant qu'espace de sociabilité et de référence pour les citadins montréalais, notamment ceux issus du Maghreb. L'on conçoit le PetitMaghreb ainsi :

«Cet espace, qui concentre assurément plus de commerces qu'il ne constitue un quartier de résidence des Maghrébins, se présente comme une des formes de la maghrébinité. Les cafés, souvent présentés comme populaires et inaccessibles aux femmes, sont un des commerces de prédilection pour les usagers du Petit-Maghreb. Ils rassemblent, de manière générale, des hommes, qui viennent retrouver leurs compatriotes. Sans être impossible, la présence des femmes se fait plus rare, tant certaines habitudes de sociabilité sont reproduites par ces dernières. La fréquentation de ces cafés illustre certaines pratiques liées au pays d'origine, mais qui cadrent avec les habitudes de vie montréalaises » (Manaï, 2014 : p. 2)

7 La réflexion quant au degré d'engagement au sein des organisations militantes ou communautaires a toujours être guidée par l'éthique et la recherche de l'équilibre. C'est 
la constante réflexivité sur les frontières sociales qui a équilibré la position du chercheur et par là-même la recherche. Seule une certaine conscience des frontières à ne pas dépasser permet de se mouvoir sur son terrain. Au final, ce contexte de militance a surtout permis de nouer des relations de confiance qui ont facilité le traitement du sujet qu'étaient les Maghrébins. Il est certain que le fait d'avoir été impliquée socialement permettait de détecter plus aisément les acteurs à rencontrer. Ainsi, les nombreuses précautions prises ont facilité le travail de chercheure et la posture de citoyenne.

8 Lors de la sortie de terrain, après la fin du terrain et après le travail doctoral, la relation à cet espace n'a pas changé. Il fallait qu'elle reste une relation de confiance, basée sur l'échange et non sur l'instrumentalisation. Des raisons éthiques m'ont ainsi conduite à refuser des interventions médiatiques sur le Petit-Maghreb ou à partir du PetitMaghreb car celles-ci n'allaient pas dans le sens des acteurs locaux. En réalisant des balades urbaines, pour des universités, des classes ou des festivals culturels, entre 2013 et 2018, la parole des commerçants ou des organismes communautaires étaient mises en avant. Ce respect vis-à-vis du terrain est primordial si l'on ne souhaite pas instrumentaliser la parole des acteurs, celle-là même qui permet aux chercheurs de produire leurs pensées et de consolider leurs carrières. Être une chercheure de l'intérieur permettait de dépasser le rôle de " fixer ${ }^{1}$ ", qu'imputent certains journalistes, universitaires ou personnalités politiques. Habiter cette position de "chercheure engagée à l'intérieur " permet de dépersonnaliser le rapport entre le chercheur et son terrain, en même temps qu'il construit le rapport de confiance et d'éviter de tomber dans la concurrence imposée par les procédures de production de la connaissance propres au milieu académique (Fontan, 2000).

C'est en saisissant l'unicité de ce regard à apporter, en restituant les paroles des acteurs d'un terrain en pleine effervescence, que le questionnement sur l'ethnicité, où la " maghrébinité » allait être un exemple et une ressource, a été travaillé grâce à un accès privilégié, notamment du point de vue linguistique. Encore fallait-il avoir ou créer sa place comme femme, dans des espaces considérés comme dédiés aux hommes.

\section{Une femme chercheure sur le "terrain des hommes" : défi et possibilités}

Bien que les femmes n'aient pas nécessairement une pratique de la ville similaire (Lévy, 2016), dans le contexte nord-américain où la ville est pratiquée de façon fluide par les hommes et les femmes, le Petit-Maghreb a fait les unes des médias à plusieurs reprises, ces derniers dénonçant l'absence des femmes dans les cafés. L'objet observé devait être travaillé avec prudence. La seule absence des femmes, dans les cafés, permettait-elle de justifier ou d'illustrer la domination masculine ou les relations patriarcales? Le fait d'être une femme a été un défi à plusieurs égards, mais la fréquentation des cafés, le fait d'entrer dans des espaces fréquentés majoritairement par des hommes, le fait d'inviter d'autres acteurs à venir faire leurs entrevues dans les espaces dits masculins, ont constitué autant de possibilités de lire l'espace autrement.

11 Dans le cas du Petit-Maghreb, il fallait prendre soin d'observer la présence factuelle des genres, à savoir " combien de femmes fréquentent les commerces? ", sans pour autant avoir de lecture culturaliste ou essentialiste. Il fallait regarder la réalité avec la 
sensibilité féministe, prônée quotidiennement en dehors du terrain, sans qu'elle empêche d'observer les autres oppressions systémiques. Il a fallu se rattacher à une perspective féministe, définie comme un moyen de se «sensibiliser au caractère situé de chaque savoir » comme l'explique Cefaï à propos des travaux de Abu Lughod (Cefaï, 2010). Chaque fois, le rôle du chercheur incitait à ne pas s'inscrire dans les convenances de genre qui s'imposent à nombre de femmes. Si les femmes n'étaient pas des clientes des cafés des hommes, notre présence dans ces mêmes espaces n'en était pas moins légitime. Il était impossible de passer outre les freins de certaines répondantes refusant des entrevues dans ces lieux, mais il était nécessaire de continuer à les fréquenter, à les observer et à y discuter.

Par cette présence en tant que chercheure, au sein d'institutions qui sont vues, à tort ou à raison comme des espaces masculins, il fallait que le genre soit dépassé. C'est en agissant ainsi, qu'il a été possible d'observer que les cafés étaient des espaces où la masculinité n'était prédominante que parce que peu de femmes se donnaient le droit d'y accéder. Ainsi, avec la patience que le terrain nous apprend à avoir, dans certains cafés, il a été possible de rencontrer des femmes de toutes origines confondues, notamment des femmes arabes et maghrébines.

La question des usages féminins du lieu se pose assurément. En effet, il a semblé, sans conteste, qu'il y avait un usage plus fluide de ces lieux. Ces femmes avaient un usage fonctionnel et utilitariste de l'espace commercial, là où certains hommes y alliaient une sociabilité. Aller chercher un café, s'installer pour manger ou prendre un dessert en disposant de l'accès à internet, voilà les usages qui semblaient être ceux des femmes.

Les raisons de cette fluidité semblent nombreuses, mais seraient imputables autant aux hommes qu'aux femmes. En effet, il est apparu que les espaces de sociabilité tels que les cafés sont autant appropriés par les hommes qu'ils ne sont pas réappropriés par les femmes. Il a été très intéressant d'observer que durant certains moments, périodes et évènements, les femmes étaient beaucoup plus présentes. L'élément majeur qui semble corroborer l'hypothèse de la mise à distance de ces lieux par les femmes elles-mêmes, consiste à observer l'usage qui en est fait par des jeunes filles, d'origine maghrébine, ayant grandi et ayant eu une socialisation à Montréal. En effet, la fréquentation du Petit-Maghreb par les jeunes filles, a priori d'origine maghrébine, portant leurs drapeaux lors des matchs de soccer, a semblé être l'argument majeur qui invite à voir la fréquentation des cafés par les femmes comme une construction sociale et une reproduction de pratiques du pays d'origine. L'idée que l'accès aux cafés était difficile aux femmes concernait en fait certaines catégories de femmes. Bien peu de lieux établissaient une frontière. En poussant la porte de certains cafés ou en souhaitant rencontrer des répondantes dans ces mêmes cafés, il était évident que la frontière entre les genres était mentale. Et le fait de pousser cette limite en s'asseyant, comme chercheure, durant des heures dans un espace qui nous plaçait en situation de minoritaire, a permis d'être parfois le sujet de notre propre recherche (Cefaï et Amiraux, 2002).

15 Au final, cette présence a fini par être une habitude pour les regards étonnés des premières fois. En fait, tout se passait comme si le fait de ne pas reconnaître une certaine présence des femmes dans ces lieux était une façon de céder à une perception normative des usages que font les hommes et les femmes. Une vision féministe de l'espace, comme le préconisent certaines géographes, implique de s'attarder sur des 
éléments qui ne sont pas constitutifs de la pratique masculine de l'espace, invitant à dépasser le dualisme :

«Wilson conçoit l'espace féministe comme un espace paradoxal, fluide, contingent. L'imagination géographique devra aider les femmes à re-conceptualiser, à revisionner l'espace. » (Chapman, 1997 : p. 16)

Durant les années de terrain, soit entre 2010 et 2014, le fait d'entendre de nombreux acteurs locaux répéter que la présence des femmes était problématique, par le fait que les cafés semblaient être fréquentés par des hommes nécessitait une réponse méthodologique. Même si l'on a choisi de ne pas dresser l'état des lieux de la fréquentation des lieux publics par les femmes, parce nous tentions de saisir les dynamiques territoriales, force est de constater que cette question, sans être centrale à l'analyse de l'espace du Petit-Maghreb, influence le terrain. Et cette influence s'opère d'autant plus parce qu'il est attendu, dans des lieux pratiqués par des immigrants, qui plus est arabes, maghrébins et musulmans, que les femmes soient présentes. Sans quoi cette absence prouverait les préjugés et représentations communes sur ces catégories de population.

17 De plus, sur ce terrain et dans la société en général, il semble attendu d'une chercheure de l'intérieur, d'adopter une position de dénonciation ou de distanciation vis-à-vis de l'objet maghrébin ou musulman, surtout s'il concerne «le garçon arabe » pour paraphraser Nacira Guénif-Souilamas et Éric Macé (2004). En instrumentalisant ainsi le rapport de genre à l'intérieur même des populations maghrébines, arabes ou musulmanes, l'on crée des lignes de fractures sociales qui ont de lourdes conséquences, comme le fait d'essentialiser le machisme ou le patriarcat. À l'instar de Mara Viveros Vigoya, il est important de se poser cette question: «Quelles conséquences peut-il y avoir à isoler une catégorie d'analyse comme le genre d'autres catégories, centrales dans les sciences sociales, comme la classe et la race?» (Viveros Vigoya, 2004:60). L'éthique de la situation d'une géographe féministe, basant ses positions sur un féminisme décolonial et sur une géographie critique (Springer, 2018), consiste donc à ne pas se résoudre à « isoler le genre » car cela se résumerait à s'isoler d'une partie de l'analyse de l'espace en plus de participer à un « racisme vertueux » comme l'explique Tissot (2005).

18 L'idée de concentrer le regard sur le fait que les femmes ne sont pas assises dans les cafés, semble éluder leur participation à d'autres niveaux d'organisation et comme autres catégories d'usagères. En effet, on pouvait noter une présence des femmes parmi toutes les salariées, qui travaillent dans les boulangeries, les pâtisseries, les agences de voyage ou encore dans certains salons de thé ou brasseries composant l'artère commerciale du Petit-Maghreb. Ensuite, il y avait toutes les clientes, d'origines diverses, qui venaient s'approvisionner dans la plupart des commerces, tels que les épiceries et les boucheries ou encore les pâtisseries. Enfin, il y a toutes les commerçantes, moins visibles dans les évènements organisés, qui sont propriétaires d'agences de voyage ou de magasins de vente de vêtements. Il y a celles qui participent très activement à l'effervescence des quartiers environnant et qui possèdent des garderies et des Centres de la Petite Enfance, devenue une niche économique (ou ethnique) pour nombre d'immigrantes originaires d'Afrique du Nord au Québec (Allali, 2010), notamment celles portant un voile, visibilisant leur "islamité", et qui ne peuvent trouver de travail ailleurs (Boudarbat, 2013). 
Donc, dans cet espace du Petit-Maghreb tel qu'il a été observé, il fallait avoir un regard prenant en compte le genre sans toutefois avoir de préjugés quant à l'usage normatif des espaces publics et commerciaux. En somme, les frontières de la définition de soi comme chercheure, à l'intersection du genre et de l'ethnicité, devaient se faire dans une fluidité constante. La possibilité de prendre sa place dans certains espaces conçus comme impossibles aux femmes nous a permis d'avoir une capacité à regarder des lieux d'un grand intérêt sociologique et urbain.

\section{Ce que l'ethnographie dans les cafés permet de révéler}

Dans les cafés, il nous a toujours été possible de trouver une place assise parmi les clients. Le premier quartier général de la recherche était le café le plus fréquenté de l'artère, le second était un nouveau café qui avait affiché un jour une pancarte indiquant "ouvert aux dames». Les va-et-vient entre ces espaces permettaient de devenir la "mouche sur le mur ", en quelque sorte, capable d'observer et de converser avec les usagers quand les opportunités d'interactions le permettaient. La présence en ces lieux permettait alors de travailler sur un espace qui était lui-même porteur de réalités très diverses. D'abord le Petit-Maghreb était un des nœuds de concentration de la « maghrébinité » montréalaise (Hardwick, 2006 ; Manaï, 2015 ; Richard, 2011). Ensuite le Petit-Maghreb rassemblait des travailleurs convertis en commerçants par les difficultés à s'insérer à un marché de l'emploi discriminatoire (Boudarbat, 2013). Enfin le Petit-Maghreb était un laboratoire pour saisir les problématiques socio-ethniques sans qu'elles soient représentatives de tous les autres espaces ${ }^{2}$ dans lesquels il y a une présence maghrébine (Manaï, 2015).

Le Petit-Maghreb, comme label institutionnalisé et nouvel objet de marketing urbain (Germain, 2013), semble donner des clés de compréhension sur la «maghrébinité " montréalaise. Ainsi, les acteurs rencontrés développaient une vision sur ces nouveaux immigrants à travers les problématiques rencontrées dans ce lieu. Par exemple, les acteurs du milieu communautaire ${ }^{3}$ et notamment les structures associatives maghrébines se penchaient sur les difficultés rencontrées par les femmes-elles mêmes : chômage, reconversions professionnelles, problématiques de violence familiale et conjugale, défis de l'immigration etc. Certains expliquaient les difficultés rencontrées par des facteurs culturels, comme cet acteur issu des communautés maghrébines :

$$
\begin{aligned}
& \text { Les femmes viennent rarement. Il y a des conflits familiaux. Il y a un taux de } \\
& \text { divorce. C'est à cause du système. La femme quand elle était en Algérie elle } \\
& \text { était soumise et ici, c'est différent. Ici elle peut travailler, elle a plus de } \\
& \text { liberté. (Acteur communautaire) }
\end{aligned}
$$

D'autres expliquaient les difficultés vécues par les femmes du fait de la religion et sa pratique dite intégriste, comme cette femme travaillant dans un organisme qui appuie les néo-Québécois ${ }^{4}$ dans leur insertion sociale :

Quand on a des activités, les femmes participent beaucoup. Et puis les femmes maghrébines par exemple peuvent éprouver des difficultés face à leurs maris, d'ailleurs souvent les mêmes que les femmes haïtiennes. Au niveau du rapport Homme-Femme, mais elles apprennent, et on leur montre leurs droits. Des fois elles viennent nous dire, oui mais l'islam, moi je dis, les 
femmes ont des droits, ce sont les intégristes qui nous les enlèvent. C'est comme toute religion, c'est comme les pasteurs qui disent les femmes ne doivent pas faire ça ou ça. Alors qu'au final c'est pas la bible, c'est juste que c'est particulièrement lui qui veut dicter sa loi. (Acteur communautaire) de comprendre pourquoi les femmes ne venaient pas dans les cafés :

J'aimerais beaucoup le savoir. J'aimerais savoir si elles se permettent de faire des trucs parallèles à ce que font les hommes. Est-ce que les hommes viennent dans les cafés pendant que les femmes restent à la maison ou les femmes aussi vont quelque part se sociabiliser. Parce que ce n'est pas l'impression que ça me donne. Ce n'est pas ce que ça me laisse. Et je me dis, encore-là avec tout le respect, j'aimerais ça les voir ces femmes-là. Parce que j'ai comme l'impression que l'on pourrait avoir une meilleure compréhension de ce qui se passe au niveau culturel, mais au niveau familial aussi. Parce que même quand on fait l'intervention policière dans les familles, souvent et presque la totalité des fois, c'est l'homme qui parle. Même quand la femme est présente et ça c'est flagrant. Et on se dit mais... Moi ça me préoccupe quand je ne peux pas avoir la version de tous les gens qui sont là. Quand je rentre et il y a une problématique ben je voudrais bien avoir ce que la maman pense. Mais très peu souvent, je ne peux pas avoir la version maternelle. Et même dans les écoles, même quand on fait une rencontre dans les écoles, c'est très peu souvent. Quand j'ai une maman qui est là, ben souvent c'est soit parce que elle est divorcée ou parce que le père est en voyage ... ou c'est parce que elle est divorcée. Ou parce que l'homme est absent. Et ça, ça vient me chercher. Ça, ça me préoccupe parce que je me rends compte aussi que j'ai beaucoup de jeunes adolescents qui ont, qui ont peu de respect aussi envers leur mère et je me dis encore là, avec un œil un peu critique. J'aimerais bien savoir d'où vient cette façon-là. Est-ce que le jeune reproduit ce qu'il voit à la maison par rapport à son père ou autre chose. Je vous dirais que ça m'interpelle mais tu sais je ne saute pas aux conclusions. Ce n'est pas ça. Mais j'dirais même cette année, j'ai vu une certaine radicalisation du Petit Maghreb. J'ai vu une boutique, j'sais pas si elle existe encore. J'ai vu une boutique qui vend des hidjabs. Non! Des niqabs. Le voile intégral. Et on voit rarement ça au Québec et là, tout d'un coup j'ai $v u$ cette boutique-là et c'est venu vraiment, vraiment m'interpeller... (Acteur institutionnel).

24

Les acteurs politiques avaient pour leur part une intention louable au demeurant, mais qui ne coïncidait pas avec les réalités socio-économiques des lieux. En effet, malgré un discours sur la reconnaissance, les termes de cette dernière n'étaient que peu définis. D'ailleurs comme peut le montrer cet extrait, cet objectif de reconnaître le PetitMaghreb devenait un peu flou : était-ce pour que les Montréalais y voient une sorte d'espace exotique? Ou était-ce à destination des Maghrébins, pour qu'ils aient un sentiment d'appartenance à la ville? Quel qu'en soit l'objectif majeur, les velléités d'égalité entre femmes et hommes, aussi symboliques soient-elles, disent beaucoup plus des attentes des acteurs sur cet espace du Petit-Maghreb : 
Je te dirai qu'on a souvent des plaintes de femmes non-maghrébines qui nous disent que les hommes ne se tassent pas pour nous laisser passer. On se fait siffler, on se sent regardées quand on passe. Est-ce que ça c'est de l'incompréhension, c'est de l'intolérance, y a aussi ça. Parce qu'on ne peut pas tout prendre non plus, nous quand on y va, ça ne nous arrive pas. On n'est peut-être pas leur genre! Je ne suis pas sûre qu'il faille tout prendre. Nous, le jour où on a eu nos toilettes au café, on a considéré que les femmes avaient leur place. Ils avaient pas de toilettes pour les femmes, puis je leur disais mais comment voulez-vous être ouverts s'il n'y a pas de toilettes pour femmes. Puis ils étaient gênés que j'y aille. Et un jour sans que je le demande, c'est lui qui m'a donné les clefs, il m'a dit va voir y a des toilettes en bas. Puis j'ai trouvé que c'était une preuve d'ouverture. Tu sais moi je ne veux pas créer de ghettos là. Je veux que tout le monde puisse venir au café. Puis avec le foot ça s'est ouvert. Les filles ne rentraient pas avant. C'est récent. Ça fait peut-être cinq ans. Maintenant les filles qui sont nées ici, elles s'en foutent, elles vont au café, mais la première fois que je suis allée au café avec A. et qu'on s'est assises, je n'étais pas encore élue, on est entrées puis on a senti un silence, on l'a bu notre café, puis on s'est dit on va revenir, on va revenir puis on va l'avoir notre place. Ça a été comme un défi personnel. Ce n'était pas de même il y a 5 ans, ça a beaucoup changé. Ce changement est dû certainement à la reconnaissance de leur statut, en leur disant vous avez le droit d'exister, vous avez le droit d'avoir un café c'est pas illégal. (Actrice politique)

Ces exemples de discours incarnent la normativité de l'espace urbain dans les usages par le genre. Force est de constater qu'en plus de voir cet espace comme le «fief des mâles " pour paraphraser le titre d'un article paru dans le journal Le Monde (Arlandis, 2012), une suspicion autour de l'altérité culturelle et religieuse s'y répand vers d'autres espaces de la vie sociale ou de la ville. L'on passe donc des femmes absentes dans les cafés des Maghrébins, aux femmes soumises dans les maisons des musulmans, traçant une ligne continue entre les espaces de consommation dans l'espace urbain et l'espace intime et privé.

Ainsi, la plupart des discours portaient sur la soumission ou l'absence des femmes. Peu en effet, admettaient une fluidité de l'usage ou encore un usage différent de l'attente des acteurs. Par ailleurs, à en croire tous les acteurs, les femmes portaient un voile dans une grande majorité. Or les observations dans les cafés et dans la rue laissaient voir le contraire. Comme le décrit cet extrait de carnet de terrain de 2012, les femmes qui ont un usage fluide de l'espace semblent être un angle mort :

En cette veille de ramadan je vais prendre le pouls de l'animation et de l'effervescence dans le quartier. Je suis arrivée aux alentours de $16: 30$ et suis restée une heure. À mon grand étonnement, les rues étaient plutôt vides. Au café italien la vie continue. Quelques personnes sont attablées avec leurs verres. Je marche dans la rue Jean talon en direction de l'est. Le coiffeur haïtien reçoit ses clients et la vie semble banale. Je décide de m'arrêter à la pâtisserie « la Table fleurie ». Juste avant de traverser la rue, un groupe de trois femmes d'environ 35 à 40 ans attire mon attention. Elles étaient audibles tant leur rires étaient forts. Elles 
se sont dirigées vers la pâtisserie et ont acheté des pâtisseries algériennes en grande quantité. Aucune d'entre elles ne portait le voile. qui avaient ouvert des commerces et étaient devenues des entrepreneures pour développer leurs propres activités et être moins à la merci des employeurs qui invoquent la nécessité du retour aux études, qui ne donnent pas d'équivalences professionnelles ou qui n'acceptent pas les femmes portant un foulard, pratique pourtant légale au Québec et Canada malgré la multitude de controverses et de débats médiatiques et politiques qui semblent dire le contraire. Car en effet, ce que l'ethnographie de la ville permet de développer, c'est un regard qui dépasse les normes et les attentes des acteurs, pour se pencher sur les réalités des individus, des groupes d'appartenance, de leur pratique spatiale et de leurs contraintes. Ainsi, travailler sur le Petit-Maghreb, impliquait également de ne pas succomber à l'apparente absence des femmes et ne pas nier les difficultés majeures des hommes. Si les postures sur l'ethnicité et le genre ont été essentielles, il n'était pas possible de faire fi des enjeux de classes et d'inégalités sociales.

\section{S'objectiver pour placer l'intersection des injustices urbaines et sociales dans un contexte d'islamophobie}

Au Québec, la scolarisation des immigrants du Maghreb, femmes et hommes, décrite par plusieurs recherches (Renaud, 2004 ; Eid, 2004; Arcand et al, 2009; Allali, 2010) aurait dû être un atout dans l'insertion économique. Si la problématique du chômage était centrale pour la plupart des acteurs maghrébins, commerçants ou issus d'organismes communautaires, il y avait une difficulté à saisir le fossé entre le niveau scolaire et l'incapacité à trouver un emploi sur le marché du travail :

Alors que les Maghrébins eux ils cherchent une reconnaissance, ils arrivent bardés de diplômes et ils commencent au bas de l'échelle. (Acteur communautaire)

Cette forme de dissonance entre la trajectoire de recrutement de ces immigrants et leur insertion réelle se retrouve au Petit-Maghreb. Ces immigrants et immigrantes devenus des entrepreneurs ont eu à se reconvertir pour contrer la déqualification (Manaï, 2018). C'est bien cette alternative que le Petit-Maghreb, comme un des espaces de la ville, semble proposer aux néo-arrivants à la recherche de " petits boulots ", aux immigrants en mal de pays ou aux nostalgiques de leur vie à Alger, à Tunis ou à Casablanca. Sans être un lieu homogène maghrébin, sans être un lieu où régnerait un ordre social maghrébin, sans être une zone d'auto-exclusion, il se définit comme un espace consolidant une référence maghrébine dans une ville plurielle et comme un espace victime de sa propre ambivalence à définir l'ethnicité qui le constitue, oscillant entre « algérianité ${ }^{5}$ » et " maghrébinité ». Il se définit surtout comme une des zones de la ville vivant une grande mutation démographique par la gentrification qui touche les environs de l'artère commerciale. Cet espace, pour lequel on peut parler de « fabrique de l'espace ethnique » en est un qui doit être observé minutieusement et sans filtre idéologique. 
30 Seule un travail éthique, commençant lors de l'approche du terrain jusqu'à sa sortie, peut décrire cet espace urbain comme il se doit d'être dit : soit dans les mots de tous les acteurs, femmes et hommes qui le font au quotidien, celles et ceux qui le vivent et le produisent, en forment les contours et les frontières. Et pour que la critique ou la théorie puissent avoir un impact sur cet espace, il s'agit de saisir dans quel contexte territorial plus large ces dynamiques s'inscrivent. Si l'on ne saisit pas l'aspect territorialisé de l'islamophobie ambiante et son empreinte sur les quartiers urbains, notamment ceux qualifiés d'ethniques, l'on passe à côté de la sensibilité que nous impose la réalité du racisme. Ainsi, comme chercheure de l'intérieur, j'ai opté pour une position engagée, où la réalité sociale est révélée par les acteurs et où la recherche devient une opportunité de transformer la narrative ou le récit sur le territoire. Par exemple, les maintes sollicitations médiatiques, dans un contexte où le Québec découvre ses immigrants du Maghreb et dans un contexte où l'islam fait l'objet de controverses et d'obsessions récurrentes, devaient être traitées avec beaucoup de précaution. Les perceptions qui règnent sur ce type d'espace nient leur complexité. Or un tel contexte, où l'ethnographie a permis de développer une succession de réflexions et d'observations, permet d'interroger la démarche de recherche et la construction de la position «d'expert». Par ailleurs, dans une ville comme Montréal où la reconnaissance de l'ethnicité dans les quartiers de la ville peut faire l'objet d'activités touristiques et ludiques, comment est-il possible de continuer en tant que chercheur à restituer la complexité tout en la confrontant aux nouvelles réalités. Or ces deux types d'interrogations qui visent à faire connaître l'espace méconnu et mal perçu, doivent impérativement comporter une part éthique qui utilise le regard du chercheur pour mieux le poser sur les acteurs, les lieux, les trajectoires, les changements et la fluidité qui continue de s'y opérer.

31 Pour reprendre les propos de Sayad, il fallait donc ici méthodiquement s'objectiver, intervenir, dire, sans que l'ethnicité du chercheur, son genre ou sa classe sociale puisse s'objecter aux réalités. Cette démarche d'objectivation constante et fluide, ne pouvait qu'admettre les sens de la présence des hommes et celui de l'absence des femmes. Or c'est bien en saisissant les enjeux différentiels qu'il est possible d'établir un propos sur l'égalité. En l'occurrence ici, les maux qui ont été projetés sur le Petit-Maghreb, les solutions qui y ont été apportées et les bilans qui y ont été réalisés semblent passer à côté de la réalité sociale et urbaine des lieux. Le Petit-Maghreb permet-il de questionner les projets urbains touristiques lorsque les individus sont ancrés dans des réalités "d'entrepreneuriat de survie " (Allali, 2010) ? Comment est-ce possible de concevoir des espaces dits ethniques en espérant qu'ils soient le plus neutrifiés ? Est-ce possible de concevoir une amélioration des services pour une clientèle dite exotique (Raulin, 2000) alors même que le marché de l'ethnique se constitue encore pour des raisons d'accès à des emplois dignes?

Que le Petit-Maghreb fasse de la place aux femmes dans les cafés ou qu'il n'en fasse pas, ne semble pas vraiment au cœur des enjeux portés par les femmes elles-mêmes. L'élargissement des réflexions que nous, chercheurs, observons sur nos terrains, surtout dans la ville, devraient se réaliser en lien avec des comparaisons spatiales qui peuvent faire émerger les différences d'usage et de pratiques. Les espaces urbains dit populaires, proposent plus souvent que nos schèmes normatifs nous permettent de le voir, un réalisme et une multitude des appartenances que seule la ville est capable de proposer. Au chercheur de faire la démarche d'objectivation pour y trouver, non ce 
qu'il voudrait y voir, mais un contexte spécifique porteur qu'il regardera à travers sa propre intersection. Il nous faut incontestablement écrire la place des femmes dans la ville, sans pour autant instrumentaliser le féminisme pour mettre à la marge l'expérience urbaine des hommes, notamment issus de groupes ethniques ou immigrants. Au Petit-Maghreb, cette écriture de l'urbanité des femmes s'accompagnait nécessairement d'une écriture des conditions sociales et urbaines partagées avec les hommes.

\section{BIBLIOGRAPHIE}

Allali, Brahim (2010) Entrepreneuriat maghrébin au Québec : un entrepreneuriat par nécessité ?, 10e Congrès international francophone en entrepreneuriat et PME, Bordeaux, Université Montesquieu Bordeaux IV, pp. 1-16.

Arcand, Sébastien ; Annick Lenoir-Achdjian ; Denise Helly (2009) Insertion professionnelle d'immigrants récents et réseaux sociaux : le cas de Maghrébins à Montréal et Sherbrooke, Canadian Journal of Sociology/Cahiers canadiens de sociologie, vol. 34, $\mathrm{n}^{\circ}$ 2, pp. 373-402.

Arlandis, Fanny (2012). La rue, fief des mâles, Le Monde, [Disponible sur Internet]

Ben Soltane, Sonia (2015) Femmes maghrébines immigrantes au Québec : une double absence, in Hamrouni, N. ; Maillé, C. (dir.) Le sujet du féminisme est-il blanc, Montréal, Éditions Remue-Ménage, pp. 209-228.

Boudarbat, Brahim ; Montmarquette, Claude (2013) Origine et sources de la surqualification dans la région métropolitaine de Montréal, Montréal, Rapport du Projet CIRANO, 116 pages.

Chapman, Rosemary (1997) L'écriture de l'espace au féminin : géographie féministe et textes littéraires québécois, Recherches féministes, vol. 10, n², pp. 13-26.

Cefaï, Daniel (2010) L'engagement ethnographique, Paris, Éditions EHESS, 640 p.

Cefaï, Daniel ; Amiraux, Valérie (2002) Les risques du métier. Engagements problématiques en sciences sociales. Partie 1, Cultures \& Conflits, vol. 47, $\mathrm{n}^{\circ}$ 3, pp. 15-48.

Eid, Paul (2004) Être arabe à Montréal : réceptions et ré-appropriations d'une identité socialement compromise, in Renaud, J. ; Germain, A. ; Leloup, X. (dir.) Racisme et discrimination : permanence et résurgence d'un phénomène inavouable, Québec, Presses de l'Université Laval, pp. 149-171.

Germain, Annick (2013) La sociologie urbaine à l'épreuve de l'immigration et de l'ethnicité : de Chicago à Montréal en passant par Amsterdam, Sociologie et société, vol. 45, n² 2, pp. 87-110.

Guénif-Souilamas, Nacira ; Macé, Éric (2004) Les féministes et le garçon arabe, La Tour d'Aigues, Editions de L'Aube, $106 \mathrm{p}$.

Hardwick W., Susan (2006) Nodal Heterolocalism and Transnationalism at the United StatesCanadian Borders, The Geographical Review, vol. 96, n² 2, pp. 212-228.

Manaï, Bochra (2018) Les Maghrébins de Montréal, Montréal, Presses de l'Université de Montréal, $146 \mathrm{p}$. 
Manaï, Bochra (2015) Le Petit-Maghreb à Montréal, entre stigmatisation et marketing, in Arcand, S. ; Germain, A. (dir.) Travailler et cohabiter, Québec, Éditions Presses de L’Université Laval. pp. 251-275.

Manaï, Bochra (2014) Le Petit Maghreb : Mise en scène de l'ethnicité maghrébine à Montréal, Vivre-Ensemble Webzine, vol. 22, $\mathrm{n}^{\circ} 75$ [Disponible sur Internet].

Mirokvasic, M. (2008) Femmes et genre dans l'étude des migrations : un regard rétrospectif, Les cahiers du CEDREF, vol. 16, pp. 33-56.

Perez, Amin (2009) Le “sens du problème” chez Sayad, Hommes et Migrations, n 1280, pp. 132-139.

Rath, Jan (2007) Tourism, ethnic diversity and the city, New York, Routledge, 241 p.

Raulin, Anne (2000) L'ethnique au quotidien. Diasporas, marchés et cultures métropolitaines, Paris, L'Harmattan, 229 p.

Renaud, Jean ; Germain, Annick ; Leloup, Xavier (2004) Racisme et discrimination. Permanence et résurgence d'un phénomène inavouable, Québec, Presses de l'Université Laval, 262 p.

Richard, Myriam (2011) Immigrants et forums Internet : les représentations de la géographie résidentielle des russophones à Montréal, Montréal, INRS-UCS, 165 p. (Mémoire de Maîtrise ; Études-Urbaines).

Sayad, Abdelmalek (1999) La double absence, des désillusions de l'émigré aux souffrances de l'immigré, Paris, Édition du Seuil, 437 p.

Tissot, Sylvie (2005) Les féministes et le garçon arabe, un livre de Nacira Guénif-Souilamas et Éric Macé, Revue Mouvements, vol. 2, n 38 [Disponible sur Internet].

Viveros Vegoya, Mara (2004) Jusqu'à un certain point, ou la spécificité de la domination masculine en Amérique latine, Revue Mouvements, vol. 1, n 31, pp. 56-63 [Disponible sur Internet].

\section{NOTES}

1. Le «fixer ", par sa connaissance du terrain et des acteurs localement ancrés, donne accès aux chercheurs, aux journalistes et facilite la collecte de données.

2. Le Petit Maghreb constitue une des formes urbaines de la maghrébinité montréalaise. À Montréal, certains quartiers ou arrondissements sont habités par des Maghrébins de façon plus importante lorsque d'autres ne sont que des artères commerciales fréquentées par les Maghrébins (Manaï, 2018).

3. Le terme communautaire dans l'espace social québécois, canadien et nord-américain évoque les organisations de la société civile, les associations, les organisations communautaires autonomes qui œuvrent dans la protection sociale, dans l'accueil des immigrants, dans l'amélioration des conditions de vie des femmes ou encore sur les enjeux du logement. Le terme n'est pas connoté comme en France où il peut signifier négativement un repli sur la communauté.

4. Les nouveaux arrivants au Québec sont souvent appelés néo-arrivants ou néo-Québécois.

5. Par algérianité, nous nous entendons les marqueurs visibles et invisibles dont les individus font usage pour évoquer leurs appartenances ethniques, culturelles, linguistiques, religieuses etc. et qui font référence au pays qu'est l'Algérie. 


\section{RÉSUMÉS}

Le présent article est une analyse de la situation du chercheur à l'intersection de ses appartenances ethniques et de genre. Les scènes se passent dans un espace montréalais désormais appelé le Petit-Maghreb et qui concentre autant d'expériences vécues que de perceptions quant à la présence des femmes et aux usages des lieux par les hommes. À partir du regard du chercheur dans une démarche ethnographique, il s'agit de dépasser les présupposés et de décrypter les enjeux de l'absence des femmes et de la réalité des hommes. Ce texte invite à regarder les espaces considérés comme lieux de la sociabilité masculine, ici mêlant consommation commerciale dite ethnique et sociabilité, comme des révélateurs des questions sociales et urbaines plus larges.

\section{INDEX}

Mots-clés : genre, relations sociales de sexe, intégration sociale, espace social, méthodologie, Maghrébins

Index géographique : Canada, Montréal

\section{AUTEUR}

\section{BOCHRA MANAİ}

Docteure en Études Urbaines de l'INRS-UCS, enseignante dans des universités montréalaises et est impliquée dans le milieu communautaire à Montréal.

bmanai@uottawa.ca 